\title{
Improvement of Painful Bortezomib-Induced Peripheral Neuropathy Following Acupuncture Treatment in a Case Series of Multiple Myeloma Patients
}

\author{
Ting Bao, MD, ${ }^{1,2}$ Lixing Lao, PhD, LAc, MD, ${ }^{2}$ Michelle Medeiros, MS, MA,
} Ruixin Zhang, $\mathrm{PhD}^{2}$, Susan G. Dorsey, RN, PhD, ${ }^{3}$ and Ashraf Badros, $\mathrm{MD}^{1}$

\begin{abstract}
Background: Bortezomib-induced peripheral neuropathy (BIPN) is a common and severe dose-limiting side effect in multiple myeloma (MM) patients. Treatment with narcotics, antidepressants, and anticonvulsants are of limited value in reducing symptoms, and they have the potential for significant side effects. Acupuncture has been reported to be effective in treating neuropathic pain. There has been limited reporting on the effect of acupuncture in treating BIPN.

Objective: The aim of this study was to report on the effect of acupuncture in treating BIPN.

Design and Patients: This study was a retrospective case series of five MM patients experiencing painful BIPN. Intervention: The patients were treated with the same acupuncture protocol on a weekly basis, adjusted to twice a week or once every 2 weeks based on response. Treatment included insertion of disposable sterile acupuncture needles, which were retained for 25 minutes, at the following bilateral ear points: shen men, point zero, and two auricular points where electro-dermal signal was detected; and bilateral body acupuncture points: LI 4, TE 5, LI 11, ST 40, and Ba Feng in the upper and lower extremities.

Main Outcome Measures: All patients were assessed for severity of painful BIPN using a 0-10 numeric pain scale, with one patient assessed using the Clinical Total Neuropathy Score, Functional Assessment of Cancer Therapy-Neurotoxicity questionnaire, and Neuropathy Pain Scale.

Results: All five patients experienced immediate pain reduction after one acupuncture treatment. Two of three patients who had more than three acupuncture treatments experienced long-lasting pain reduction and function improvement. There were no adverse events associated with acupuncture.

Conclusion: Acupuncture is a viable treatment option for MM patients experiencing painful BIPN. However, further prospective research is needed.
\end{abstract}

Key Words: Acupuncture, Bortezomib, Peripheral Neuropathy, Multiple Myeloma

\section{INTRODUCTION}

T HE PROTEOSOME INHIBITOR BORTEZOMIB is an effective and widely used agent in treating multiple myeloma (MM). Bortezomib-induced peripheral neuropathy (BIPN) is one of the most common and severe toxicities of borte- zomib resulting in dose reduction or drug discontinuation. ${ }^{1}$ BIPN usually occurs within the first five cycles of bortezomib; improvement in symptoms have been reported between 3 and 48 months after the discontinuation of the drug. ${ }^{1}$ In one study, however, only $31 \%$ (11/35) of patients experiencing grade 3 BIPN had complete symptom resolution after

\footnotetext{
${ }^{1}$ The University of Maryland School of Medicine Marlene and Stewart Greenebaum Cancer Center, Baltimore, Maryland.

${ }^{2}$ The University of Maryland School of Medicine Center for Integrative Medicine, Baltimore, Maryland.

${ }^{3}$ The University of Maryland School of Nursing, Baltimore, Maryland.
} 
discontinuing bortezomib. ${ }^{2}$ There are two types of BIPN: acute painful BIPN and chronic non-painful BIPN. Painful BIPN accounts for 5-10\% of BIPN, and usually occurs suddenly within the first three cycles of bortezomib. It tends to be more severe than non-painful PN, and has not been well characterized by the National Cancer Institute Common Toxicity Criteria (NCI/CTC). Additionally, it usually takes more time to resolve after discontinuation of bortezomib. ${ }^{2,3}$ A prospective clinical trial studying nerve conduction suggests that BIPN is a length-dependent, sensory, axonal, large-fiber polyneuropathy. ${ }^{4}$ The clinical characteristics of BIPN are consistent with dysfunction of sensory nerves causing spontaneous pain, parenthesia (tingling, numbness), hyperalgesia (increased sensitivity to painful stimuli), allodynia (hypersensitivity to non-painful stimuli), and decreased physical activity. ${ }^{5}$ The treatment for BIPN has been limited to symptom management with narcotics, antidepressants, anticonvulsants, and vitamins. ${ }^{1}$ Studies suggest that such analgesic regimens usually only produce modest pain relief and are associated with side effects such as dizziness, sedation, dry mouth, and constipation. ${ }^{1,3,6}$ Therefore, more effective and less toxic treatments for BIPN are needed.

The efficacy of acupuncture for treating diabetic peripheral neuropathy and HIV-related peripheral neuropathy has been reported in several observational and pilot clinical studies. ${ }^{7-9}$ Electroacupuncture has been used as an adjunctive treatment to control neuropathic pain in cancer patients. ${ }^{10}$ One randomized, double-blinded trial established an analgesic effect for auricular acupuncture in treatment of cancer treatmentinduced neuropathy. ${ }^{11}$ A case series suggested potential efficacy of acupuncture in treating patients with chemotherapyinduced peripheral neuropathy (CIPN). ${ }^{12}$ However, to date, no clinical trial has been conducted to study acupuncture in MM patients. ${ }^{5}$ Here, we report a case series of five multiple myeloma patients suffering from BIPN treated with a standard acupuncture protocol.

\section{METHODS}

This is a retrospective chart review of five consecutive MM patients suffering from persistent painful BIPN after bortezomib discontinuation who were not responding to analgesic medications. They were treated with acupuncture at the University of Maryland Marlene and Stewart Greenebaum Cancer Center (UMGCC) between December 2009 and February 2011. This study was approved by the Institutional Review Board of the UMGCC.

Patients were assessed for severity of PN using NCI CTC and a 0-10 numeric pain scale for painful PN. One patient, in an attempt to define symptoms and response to therapy better, was assessed using Clinical Total Neuropathy Score (TNSc), Functional Assessment of Cancer Therapy/ Gynecologic Oncology Group-Neurotoxicity questionnaire (FACT/GOG-Ntx), and Neuropathy Pain Scale (NPS). The
TNSc combines information from symptoms and signs of neuropathy with sensory tests to generate a single score to quantify neuropathy. ${ }^{13}$ TNSc has demonstrated reliability and validity with inter- and intra-reliability being 0.966 and 0.986 respectively. ${ }^{13}$ The FACT/GOG-Ntx is an 11-item neurotoxicity subscale covering sensory neuropathy, motor neuropathy, hearing neuropathy, and dysfunction associated with neuropathy to assess the details of neurotoxicity symptoms in the patient. It correlates with meaningful clinical changes in neuropathy and has demonstrated reliability and validity. ${ }^{14}$ The Neuropathic Pain Scale (NPS) is a multidimensional tool that uses self-reported visual analogue scales (VAS) to quantify, on a scale of 0-10, global pain intensity and unpleasantness and eight other descriptive qualities of neuropathic pain. The NPS also includes one semi-structured question about temporal sequence that can distinguish neuropathic pain from non-neuropathic pain. ${ }^{15-17}$

Acupuncture treatment was provided on a weekly basis with adjustments of frequency based on response. The patients were informed of the risks (local pain and bruising, minor bleeding, and lightheadedness) and signed consent for the acupuncture procedure. Treatment used a standard acupuncture protocol, which includes acupoints that were selected based on our clinical experience and prior research. ${ }^{11,12}$ These included bilateral ear points (shen men, point zero, and two additional auricular acupuncture points where electro-dermal signal was detected), bilateral body acupuncture points LI 4, TE 5, LI 11, ST 40, and Ba Feng (Fig. 1). For auricular points, the electro-dermal signal was detected through a handheld auricular acupoint finder (the Pointer-Excell II ${ }^{\mathrm{TM}}$, Mayfair Medical Supplies, LTD., Hong Kong) through which an alert sounded when the electrodermal signal was detected. The procedure was performed in a quiet room using a comfortable bed or massage table. The patient was placed in a supine position. The skin at the site of acupoint was disinfected with an alcohol swab. Disposable sterilized acupuncture needles (Seirin ${ }^{\circledR}$, Japan) were used-filiform $0.16 \mathrm{~mm} \times 15 \mathrm{~mm}$ for the ear points and $0.25 \mathrm{~mm} \times 40 \mathrm{~mm}$ for the body points. The acupuncture needles were inserted 0.5 inch into the skin and remained in position for 20 minutes after the De Qi sensation - a feeling of soreness, numbness, and distention-was achieved.

\section{RESULTS}

The patients' baseline characteristics are summarized in Table 1. There were five MM patients (three male, two female) with a median age of 42 years (range: $36-57$ years). All had at least grade 2 BIPN. One patient had NCI-CTC grade 2, which interferes with function but not daily living activities; three patients had grade 3 , which is symptomatic and interferes with daily living activities; and one patient had grade 4, sensorimotor neuropathy. All patients experienced pain that 


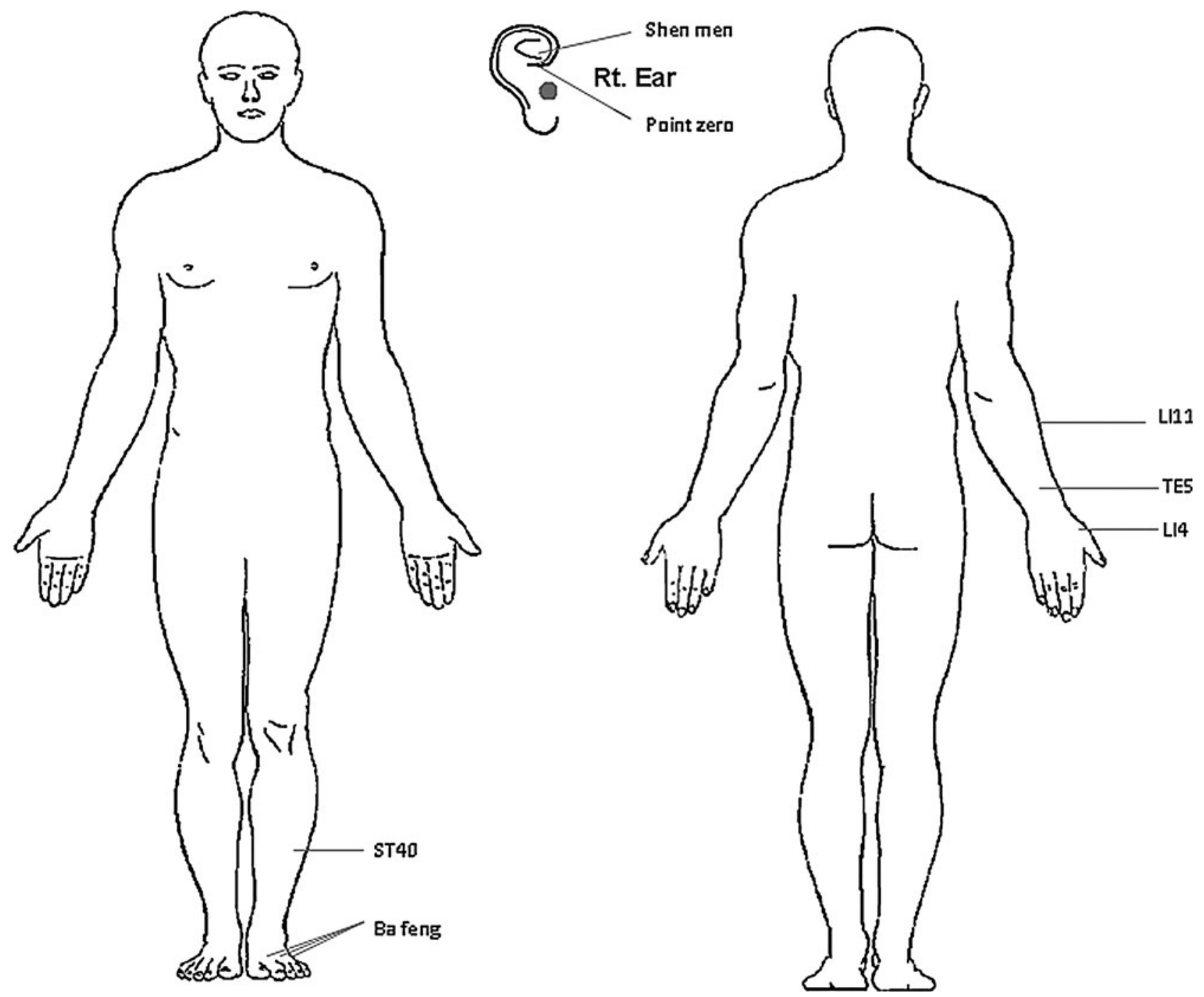

FIG. 1. Acupuncture point location map. Note: Acupuncture needles were inserted $0.5-2$ inches into the skin to obtain the De Qi sensation, and they remained in place for 20 minutes.

occurred within three cycles of bortezomib; the patients' mean pain score was 6.3 out of 10 (range: $4-10$ ).

All patients were African-American patients, a population that we have reported to have a higher incidence of painful BIPN than Caucasians. ${ }^{3}$ None of the patients had diabetes, a known risk factor for BIPN. One patient had baseline peripheral neuropathy before bortezomib was initiated. The duration of BIPN symptoms before acupuncture treatment ranged from 3 months to 12 months (median 10 months). Tingling, numbness, and pain of the lower extremities were the chief complaints at initiation of acupuncture.

Patients' treatment schedules and responses are summarized in Table 2. All five patients experienced immediate pain relief after acupuncture treatment. Two patients had to

Table 1. Summary of Patients' Baseline Characteristics

\begin{tabular}{llccccc}
\hline Patient & Age & Gender & Bortezomib: cycle, $\mathrm{mg} / \mathrm{m}^{2}$ & BIPN duration (months) & Pain score & NCI CTC grade \\
\hline 1 & 36 & M & Cy 1-3 (1.3) & 12 & 4 & 2 \\
2 & 48 & M & Cy 1-3 (1.3) & 4 & 3 & 3 \\
3 & 57 & F & Cy 1,2 (1.3) & 3 & 12 & 4.5 \\
4 & 57 & M & Cy 1-3 (1.3) & 10 & 5 \\
5 & 48 & F & Cy 1-3 (1.3) & & 3 \\
\hline
\end{tabular}


Table 2. Summary of Patients' Responses to Acupuncture Treatment

\begin{tabular}{|c|c|c|c|c|}
\hline Patient & $\begin{array}{l}\text { Pain score before } \\
\text { 1st acupuncture }\end{array}$ & $\begin{array}{l}\text { Pain score after } \\
\text { 1st acupuncture }\end{array}$ & Acupuncture treatment schedule & $\begin{array}{l}\text { Treatment response after at least } \\
\text { three acupuncture treatments }\end{array}$ \\
\hline 1 & 4 & 1 & $\begin{array}{l}3 \text { weekly acupuncture then lost to } \\
\text { follow-up for social reasons }\end{array}$ & N/A \\
\hline 2 & 8 & 0 & $\begin{array}{l}6 \text { weekly, then } 4 \text { q } 2 \text { week, then } 2 \text { q } 3 \\
\text { week, then } 2 \text { q month acupuncture } \\
\text { (total } 14 \text { treatments) }\end{array}$ & $\begin{array}{l}\text { Pain-free, no longer taking } \\
\text { narcotics, able to go back } \\
\text { to work }\end{array}$ \\
\hline 3 & 10 & 3 & $\begin{array}{l}7 \text { weekly acupuncture then stopped due } \\
\text { to lack of efficacy }\end{array}$ & No significant difference \\
\hline 4 & 4.5 & 1 & $\begin{array}{l}1 \text { acupuncture then unable to come } \\
\text { back because of transportation issues }\end{array}$ & N/A \\
\hline 5 & 5 & 0 & $\begin{array}{l}2 \text { weekly, } 4 \text { biweekly, } 3 \text { weekly, } 3 \text { q } 2 \\
\text { week, } 2 \text { q month acupuncture (total } \\
14 \text { treatments) }\end{array}$ & $\begin{array}{l}\text { Minimal pain and parenthesia, } \\
\text { improved function }\end{array}$ \\
\hline
\end{tabular}

stop acupuncture treatment after one and three sessions respectively due to transportation and social reasons (Patient 4 and Patient 1). Two of three patients who had more than three acupuncture treatments experienced long-lasting pain relief and improvement of function. The one patient who had grade 4 BIPN and with 10/10 severe pain had immediate relief after acupuncture treatment, but this did not last (Table 2). There were no adverse events associated with the acupuncture treatments.

\section{Detailed History of Patient 5}

The patient was a 48-year-old African-American woman with MM who presented with headache and fatigue. Her past medical history was significant for hypertension. She received induction therapy with bortezomib $1.3 \mathrm{mg} / \mathrm{m}^{2} \mathrm{IV}$ on days $1,4,8$, and 11 , thalidomide $200 \mathrm{mg}$ PO daily, and dexamethasone $40 \mathrm{mg}$ PO on days of bortezomib. Cycles were repeated every 21 days. After three cycles, she achieved near complete remission and underwent autologous peripheral blood stem cell transplantation. She achieved complete remission. After the third cycle of bortezomib and thalidomide, she developed grade 2 BIPN with tingling, numbness, and severe burning pain in her feet below the ankle. Her symptoms worsened despite being off bortezomib and thalidomide therapy. She reported that her painful neuropathy was so bad that "sometimes she had to sit in the bed, rub her feet and cry." She was started on pregabalin in escalating doses up to $200 \mathrm{mg}$ every 8 hours, duloxetine $60 \mathrm{mg}$ once per day, and oxycodone $5 \mathrm{mg}$ one to two tablets every 6 hours as needed for pain with no improvement. She was then switched from pregabalin to gabapentin $1200 \mathrm{mg}$ three times a day and continued on duloxetine and oxycodone and still no improvement. Her BIPN symptoms persisted with pain, tingling, numbness, and poor quality of life from neuropathic pain and pain medications. Ten months after her initial BIPN symptoms presented, she was referred for acupuncture treatment.

During the patient's initial visit to the UMGCC Acupuncture Clinic, she had NCI-CTC grade 3 peripheral neuropathy. As a result, she was unable to walk long distances, drive, or exercise. Her self-reported overall pain score was 5 out of 10 on an 11-point numeric pain scale. Her TNSc score was 9 out of 28 with sensory symptoms extended to the ankle, slight difficulty in motor function, and reduced pin and vibration sensibility to the bilateral ankles. Her muscle strength was normal but ankle reflex was absent. Her FACT/GOG-TNx score was 14 out of 44 with numbness, tingling, and constant discomfort in her feet, significant trouble walking, and occasional joint pain or muscle cramps. Her NPS score was 51 out of 100.

Immediately following her first acupuncture treatment, the patient's TNSc score decreased from 9 to 5 with her sensory symptoms and ankle reflex slightly recovered, and her pin and vibration sensibility no longer involving her ankle. Her total NPS score went down to 18 . She was treated

Table 3. Response to Acupuncture Treatment Assessed by the Functional Assessment of Cancer

Therapy/Gynecologic Oncology Group-Neurotoxicity Questionnaire (FACT/GOG-NTx), the Neuropathy Pain Scale (NPS), and Clinical Total Neuropathy Score (TNSc) in Patient 5

\begin{tabular}{|c|c|c|c|c|c|c|c|c|c|c|c|}
\hline & $9 / 3 / 10$ & $9 / 7 / 10$ & $9 / 10 / 10$ & $9 / 14 / 10$ & $9 / 17 / 10$ & $9 / 24 / 10$ & $10 / 1 / 10$ & $10 / 8 / 10$ & $11 / 5 / 10$ & $1 / 7 / 11$ & $2 / 4 / 11$ \\
\hline FACT/GOG-Ntx (0-44) & 14 & 12 & 6 & 4 & 6 & 7 & 8 & 6 & 4 & 3 & 2 \\
\hline NPS $(0-100)$ & 51 & 38 & 21 & 11 & 17 & 28 & 16 & 15 & 10 & 8 & 8 \\
\hline TNSc (0-28) & 9 & 8 & 3 & 3 & 4 & 3 & 2 & 3 & 4 & 5 & 2 \\
\hline
\end{tabular}




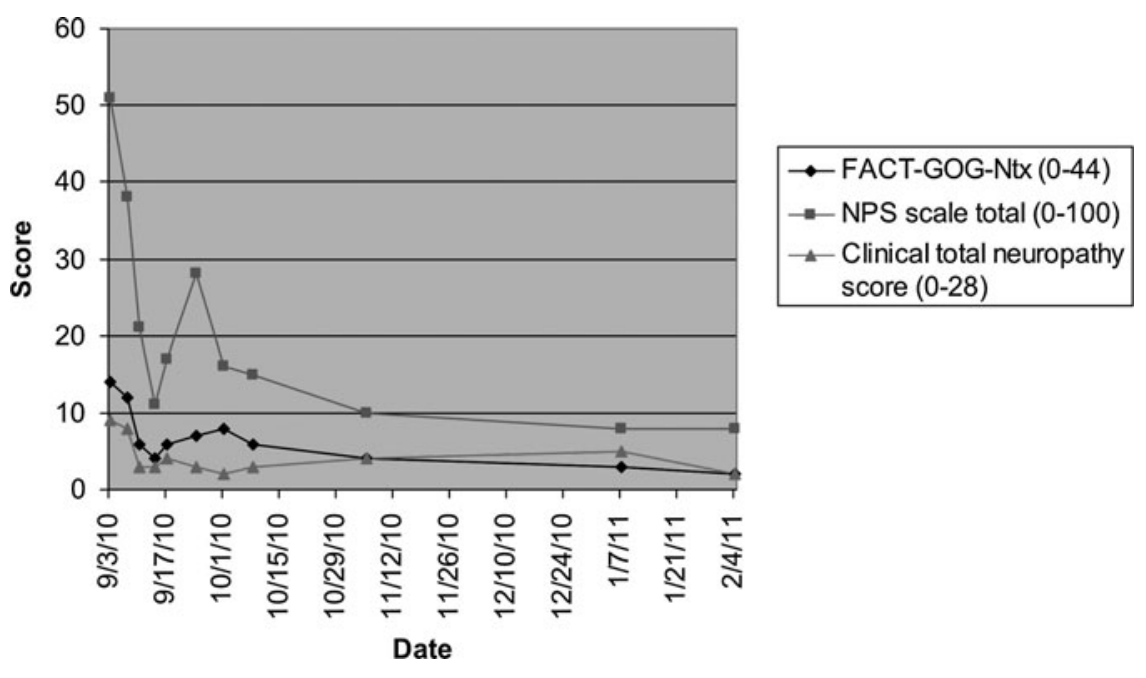

FIG. 2. Response to acupuncture treatment assessed by the Functional Assessment of Cancer Therapy/Gynecologic Oncology GroupNeurotoxicity questionnaire, the Neuropathy Pain Scale, and Clinical Total Neuropathy Score in Patient 5.

initially weekly for 2 weeks, which provided pain relief for approximately 2 days after each session. We then increased the treatment frequency to twice per week for 2 weeks, and she started noticing progressively longer responses with improvement in all end points. We then spaced out her acupuncture treatment to once per week for 3 weeks, then once every 2 weeks for 6 weeks, and finally 2 once-monthly treatments. After a total of 14 treatments, her TNSc score was reduced from 9 to 2, her NPS was reduced from 51 to 8 and FACT/GOG-TNx was lowered from 14 to 3 (Table 3 and Fig. 2). Throughout her 14 acupuncture treatments, no adverse events related to acupuncture were noted.

During the acupuncture treatments, she continued taking gabapentin $1200 \mathrm{mg}$ three times a day and duloxetine $60 \mathrm{mg}$ once per day. She was able to discontinue her breakthrough pain medication (oxycodone). At the end of the treatment, she no longer had difficulty walking and standing. She has been followed every 3 months and reported no worsening symptoms at the last follow-up, which was at 1 year after the initiation of acupuncture treatment.

\section{DISCUSSION}

This is the first case series on the use of acupuncture in treating multiple myeloma patients with BIPN. This is also the first case series using TNSc, FACT/GOG-NTx, and NPS to assess symptom severity and treatment response. Our report suggests that acupuncture is a viable treatment option for patients experiencing BIPN, especially those with grade 2 and 3 neuropathy. All patients experienced immediate pain relief after the first acupuncture treatment, and two out of three patients who had more than three acupuncture treatments had long-lasting effects. One such patient has been reported elsewhere. ${ }^{18}$
Acupuncture treatment was provided on a weekly basis with adjustments of frequency based on response. The number of acupuncture treatments was determined by the patients' availability and response. All patients were treated by the same acupuncture protocol regarding point selection and length of each acupuncture treatment (20 minutes), which was developed from our clinical experience and prior chemotherapy-induced peripheral neuropathy acupuncture research. ${ }^{11,12}$ We selected the ear points based on a randomized, blinded, controlled trial demonstrating the effectiveness of auricular acupuncture in treating cancer therapy-induced neuropathy. ${ }^{11}$ This study showed that in the group that received real auricular acupuncture (vs. placebo), pain intensity assessed using a visual analog scale decreased by $36 \%$ at the end of 2 months when compared with baseline, whereas it only decreased by $2 \%$ in the placebo groups $(P<0.0001) .{ }^{11}$ We selected the body acupuncture points based on a case series showing the efficacy of acupuncture in treating patients with chemotherapy-induced peripheral neuropathy (CIPN). ${ }^{12}$ In this case series, five patients with greater than World Health Organization grade II CIPN were treated with six weekly acupuncture treatments followed by 4 weeks of rest and six additional weekly acupuncture treatments. All but one patient finished all 12 acupuncture treatments. All five patients reported improvement in pain, tingling, and numbness. Pain scores decreased from 6-9 out of 10 to $2-3$ out of 10 after acupuncture treatments. All patients had reduced analgesic medication intake. Four out of five patients reported persistent symptom relief at the 6-month follow-up. ${ }^{12}$

We observed effects in our case series similar to those aforementioned reports, suggesting that acupuncture may be effective in relieving BIPN symptoms. Although studies have not been able to explain fully the mechanism of acupuncture, it has been proposed that acupuncture works 
through its effect on neurotransmitters and neurohormones. Animal research suggests that acupuncture accomplishes its analgesic effect by stimulating nerves in the muscle, which then relay the signal to the spinal cord, midbrain, and hypothalamus-pituitary system, which then leads to the release of neurotransmitters and hormones such as endorphins and enkephalins. ${ }^{19-21}$ Other mechanisms such as activation of descending pain-inhibiting pathways, deactivation of the limbic system, as well as cortical and subcortical regions, cortical cerebral vasodilation causing release of neuropeptide, and inhibition of the release of inflammatory factors have also been suggested to explain the effect of acupuncture analgesia. ${ }^{22-26} \mathrm{~A}$ recent study also showed that electroacupuncture alleviates bone cancer pain by suppressing spinal expression of interleukin- $1 \beta$ in glial cells that are involved in the spinal transmission and processing of noxious inputs from the peripheral sites and facilitates pain. ${ }^{27}$ It is conceivable that acupuncture reduces BIPN symptoms by suppressing activities of glial cells and stimulating neurohormonal pathways, which increases endorphine release and reduces proinflammatory cytokines. ${ }^{28}$ Lastly, animal studies suggest that acupuncture might stimulate nerve growth factors and accelerate nerve regeneration. ${ }^{29,30}$ Further study needs to be conducted to explore the role of acupuncture in helping patients suffering from BIPN and its mechanisms of action.

Our study is limited for being a small sample sized retrospective study. In addition, the patient's relief of peripheral neuropathy symptoms may be due to a spontaneous remission rather than the effect of acupuncture. However, based on our clinical experience, once BIPN becomes so severe that it interferes with daily activities, it rarely resolves by itself. And even so, it usually takes a long time (months to years) to do so.

Our case series is unique in that we use TNSc, FACT/GOGNTx, and NPS to assess one patient's response to acupuncture in addition to using the $0-10$ numeric pain scale that was used by prior acupuncture CIPN studies. By doing so, we not only report how acupuncture changed the patient's pain, but also capture the effect of acupuncture in reversing peripheral neuropathy through changes in neurological exams, patient's function, and different aspects of pain (dull, sharp, hot, cold, deep, or superficial pain). Our case series suggests the feasibility of using scales like TNSc, FACT/GOG-NTx, and NPS to assess the severity of BIPN and response to acupuncture in patients. We are using these scales and neurological tests as end points to conduct a pilot clinical trial to study the effect of acupuncture to treat BIPN and to explore the mechanism of acupuncture through correlative studies. Moreover, our case series shows that two patients no longer needed acupuncture treatment and remain pain free with improved function. This suggests that acupuncture is a long-term potential curative therapy for BIPN in addition to providing temporal relief. The role of acupuncture as preventative modality to allow cancer patients to continue neurotoxic chemotherapy merits further investigation.

\section{CONCLUSIONS}

Acupuncture is a viable adjunctive treatment option for MM patients experiencing painful BIPN. A prospective study on acupuncture for BIPN symptoms is currently underway with promising results.

\section{ACKNOWLEDGMENTS}

TB is a Paul Calabresi scholar (K12 CA126849 A). RZ and LL are supported by grant number AT004113 from the National Center for Complementary and Alternative Medicine (NCCAM) at the National Institutes of Health. SGD is supported by P30NR011396 University of Maryland, Baltimore (UMB) Center for Pain Studies.

\section{DISCLOSURE STATEMENT}

No competing financial interests exist.

\section{REFERENCES}

1. Argyriou AA, Iconomou G, Kalofonos HP. Bortezomib-induced peripheral neuropathy in multiple myeloma: a comprehensive review of the literature. Blood. 2008;112:1593-1599.

2. Richardson PG, Briemberg H, Jagannath S, et al. Frequency, characteristics, and reversibility of peripheral neuropathy during treatment of advanced multiple myeloma with bortezomib. J Clin Oncol. 2006;24:3113-3120.

3. Badros A, Goloubeva O, Dalal JS, et al. Neurotoxicity of bortezomib therapy in multiple myeloma: a single-center experience and review of the literature. Cancer. 2007;110:1042-1049.

4. Chaudhry V, Cornblath DR, Polydefkis M, Ferguson A, Borrello I. Characteristics of bortezomib- and thalidomide-induced peripheral neuropathy. J Peripher Nerv Syst. 2008;13:275-282.

5. Zhou Y, Garcia MK, Chang DZ, et al. Multiple myeloma, painful neuropathy, acupuncture? Am J Clin Oncol. 2009;32:319-325.

6. Cata JP, Weng HR, Burton AW, Villareal H, Giralt S, Dougherty PM. Quantitative sensory findings in patients with bortezomib-induced pain. J Pain. 2007;8:296-306.

7. Zhang C, Ma YX, Yan Y. Clinical effects of acupuncture for diabetic peripheral neuropathy. J Tradit Chin Med. 2010;30:13-14.

8. Jiang H, Shi K, Li X, Zhou W, Cao Y. Clinical study on the wrist-ankle acupuncture treatment for 30 cases of diabetic peripheral neuritis. J Tradit Chin Med. 2006;26:8-12.

9. Phillips KD, Skelton WD, Hand GA. Effect of acupuncture administered in a group setting on pain and subjective peripheral neuropathy in persons with human immunodeficiency virus disease. J Altern Complement Med. 2004;10:449-455.

10. Minton O, Higginson IJ. Electroacupuncture as an adjunctive treatment to control neuropathic pain in patients with cancer. J Pain Symptom Manage. 2007;33:115-117.

11. Alimi D, Rubino C, Pichard-Leandri E, Fermand-Brule S, Dubreuil-Lemaire ML, Hill C. Analgesic effect of auricular 
acupuncture for cancer pain: a randomized, blinded, controlled trial. J Clin Oncol. 2003;21:4120-4126.

12. Wong R, Sagar S. Acupuncture treatment for chemotherapyinduced peripheral neuropathy—a case series. Acupunct Med. 2006;24:87-91.

13. Cornblath DR, Chaudhry V, Carter K, et al. Total neuropathy score: validation and reliability study. Neurology. 1999;53:16601664.

14. Calhoun EA, Welshman EE, Chang $\mathrm{CH}$, et al. Psychometric evaluation of the functional assessment of cancer therapy/ gynecologic oncology group-neurotoxicity (FACT/GOG-Ntx) questionnaire for patients receiving systemic chemotherapy. Int J Gynecol Cancer. 2003;13:741-748.

15. Galer BS, Jensen MP. Development and preliminary validation of a pain measure specific to neuropathic pain: the neuropathic pain scale. Neurology. 1997;48:332-338.

16. Jensen MP, Friedman M, Bonzo D, Richards P. The validity of the neuropathic pain scale for assessing diabetic neuropathic pain in a clinical trial. Clin J Pain. 2006;22:97-103.

17. Fishbain DA, Lewis JE, Cutler R, Cole B, Rosomoff HL, Rosomoff RS. Can the neuropathic pain scale discriminate between non-neuropathic and neuropathic pain? Pain Med. 2008;9:149-160.

18. Bao T, Zhang R, Badros A, Lao L. Acupuncture treatment for bortezomib-induced peripheral neuropathy: a case report. Pain Res Treat. 2011. doi: 10.1155/2011/920807.

19. Han JS. Acupuncture: neuropeptide release produced by electrical stimulation of different frequencies. Trends Neurosci. 2003;26:17-22.

20. Berman B. A 60-year-old woman considering acupuncture for knee pain. JAMA. 2007;297:1697-1707.

21. Li A, Lao L, Wang Y, et al. Electroacupuncture activates corticotrophin-releasing hormone-containing neurons in the paraventricular nucleus of the hypothalammus to alleviate edema in a rat model of inflammation. BMC Complement Altern Med. 2008;8:20

22. Hui KK, Liu J, Marina O, et al. The integrated response of the human cerebro-cerebellar and limbic systems to acupuncture stimulation at ST 36 as evidenced by fMRI. Neuroimage. 2005;27:479-496.

23. Jansen G, Lundeberg T, Kjartansson J, Samuelson UE. Acupuncture and sensory neuropeptides increase cutaneous blood flow in rats. Neurosci Lett. 1989;97:305-309.

24. Kashiba H, Ueda Y. Acupuncture to the skin induces release of substance $\mathrm{p}$ and calcitonin gene-related peptide from peripheral terminals of primary sensory neurons in the rat. Am J Chin Med. 1991;19:189-197.

25. Leong RJ, Chernow B. The effects of acupuncture on operative pain and the hormonal responses to stress. Int Anesthesiol Clin. 1988;26:213-217.

26. Li A, Wang Y, Xin J, et al. Electroacupuncture suppresses hyperalgesia and spinal Fos expression by activating the descending inhibitory system. Brain Res. 2007;1186:171-179.

27. Zhang RX, Li A, Liu B, et al. Electroacupuncture attenuates bone cancer pain and inhibits spinal interleukin-1 beta expression in a rat model. Anesth Analg. 2007;105:1482-1488.

28. Chae Y, Hong MS, Kim GH, et al. Protein array analysis of cytokine levels on the action of acupuncture in carrageenaninduced inflammation. Neurol Res. 2007;29:S55-58.

29. La JL, Jalali S, Shami SA. Morphological studies on crushed sciatic nerve of rabbits with electroacupuncture or diclofenac sodium treatment. Am J Chin Med. 2005;33:663-669.

30. Chen YS, Yao $\mathrm{CH}$, Chen $\mathrm{TH}$, et al. Effect of acupuncture stimulation on peripheral nerve regeneration using silicone rubber chambers. Am J Chin Med. 2001;29:377-385.

Address correspondence to:

Ting Bao, $M D$

Assistant Professor of Oncology

Marlene and Stewart Greenebaum Cancer Center University of Maryland School of Medicine 22 South Greene Street Baltimore, MD 21201

E-mail: tbao@umm.edu 